RICYDE. Revista Internacional de Ciencias del Deporte doi: $10.5232 /$ ricyde

Rev. int. cienc. deporte

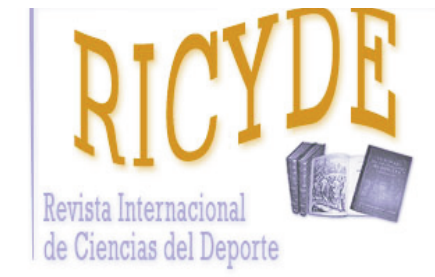

RICYDE. Revista Internacional de Ciencias del Deporte VOLUMEN XIII - AÑO XIII

Paginas:426-441 ISSN:1885-3137

Número 50 - Octubre - 2017

\title{
La lógica interna en Los Juegos de niños (1560) de Peter Brueghel Internal motricity logic in Children's Games (1560) by Peter Brueghel
}

\author{
Irene Andújar, Jordi Brasó
}

Universidad de Barcelona. España

\begin{abstract}
Resumen
El análisis del folclore y las tradiciones de una sociedad es una herramienta indispensable para dibujar y comprender un lugar y una época. En el ámbito lúdico, los juegos de la población tienen que permitir estudiar diferentes aspectos sociales tales como la tipología de estas prácticas y sus participantes, su simbolización o la situación económica de un pueblo y una clase social. El objeto de estudio del presente artículo es la obra pictórica de Peter Brueghel, Juegos de niños (1560). Concretamente, se analizan las diferentes prácticas lúdicas en función de su carácter motriz, de su lógica interna y de la utilización de algún instrumento. La metodología se ha basado en el análisis estadístico de los juegos que aparecen en el grabado y en el tratamiento de las principales fuentes textuales que interpretan el cuadro y lo relacionan con la sociedad del momento. Los resultados se relacionan y reafirman los estudios históricos de la época en los cuales se destaca la poca intervención-control por parte del adulto y unas prácticas lúdicas, mayoritariamente de bajo nivel socio-cognitivo-motriz. Se concluye que la obra permite describir la situación de los juegos en su época, vista desde la óptica del pintor, lo que tiene que permitir aplicar estas prácticas al siglo XXI, comparándolas y relacionándolas con los juegos de hoy en día.
\end{abstract}

Palabras clave: Peter Brueghel; Juegos de Niños; lógica interna, motricidad; pintura, s. XVI.

\begin{abstract}
The analysis of folklore and traditions of a society is an indispensable tool to draw and understand a place and a time. In the play area, the games of the population must allow to study different social aspects such as the typology of these practices and their participants, their symbolization or the economic situation of a people and a social class. The object of study of the present article is the pictorial work of Peter Brueghel, Games of children (1560). Specifically, we analyze the different ludic practices according to their motive nature, their internal logic and the use of some instrument. Methodology has been based on the statistical analysis of the games that appear in the engraving and in the treatment of the main textual sources that interpret the picture and relate it to the society of the time. Results reaffirm the historical studies of the time in which the low intervention-control on the part of the adult is emphasized and a playful practice, mainly of low socio-cognitive-motor level. It is concluded that the work allows to describe the situation of the games in his time, seen from the perspective of the painter, which should allow to apply these practices to the 21st century, comparing them and relating them to the games of today.
\end{abstract}

Key words: Peter Brueghel; Children's Game; internal logic motricity; painting; s. XVI.

Correspondencia/correspondence: Jordi Brasó

Universidad de Barcelona. España

Email: jbrasorius@ub.edu 
Andújar, I., y Brasó, J. (2017). La lógica interna en Los Juegos de niños (1560) de Peter Brueghel. RICYDE. Revista internacional de ciencias del deporte, 50(13), 426-441.

\section{Introducción}

L os estudios de los deportes y los juegos en las ciencias sociales pueden implicar conocimientos transferibles al entendimiento de la sociedad. Así, todo el proceso civilizador surgido a partir del s. XVI, con la aparición de modelos reglamentados en la conducta y una mayor sensibilización, quedó bien mostrado en la evolución de los deportes y los juegos. Son ejemplos de este esfuerzo civilizador, "la 'deportivización' de los pasatiempos... y la exportación de algunos de ellos a casi todo el mundo" (Elias y Dunning, 1992, 34). Aparece, por lo tanto, un proceso de control con claras similitudes a la pedagogización lúdica en la escuela de los s. XVII y XVIII. El principal objetivo de todo ello será el de reglamentar, pacificar y civilizar la conducta humana y la sociedad.

Analizando estas ideas en las representaciones artísticas, concretamente en los grabados y cuadros, se comprueban estos hechos. Un claro ejemplo de la conveniencia social, regulación y control del juego se encuentra en la obra de Hieronymus Bosch -el Bosco- (c. 1450-1516), El Jardín de las Delícias (c. 1480-1490) donde los jugadores tienen un castigo infernal, en una época donde la avaricia y el vicio son penados y, en general, cualquier actividad sujeta al azar (Gómez, 2009; Mateo, 1959, 1991). Asimismo, el tratamiento de los juegos en los grabados nos lleva a diferentes representaciones, y permite interpretar la importancia de estas prácticas -y las inquietudes- de sus creadores en la sociedad del momento. Así por ejemplo destacan las obras de: Michelangelo Merisi da Caravaggio (1571-1610) y Jugadores de cartas (1595); Antoine Le Nain (1599-1648) y Los Jugadores de trictrac (1650); Antonio de Pereda (1611-1678) y El sueño del caballero (1670); o las impresiones de la vida en la calle de Francisco de Goya (1746-1828) con Jugadores de naipes (1778), Los zancos (1778), El juego de pelota a pala (1779) o el esbozo El loco de patines (1824-28). De todas estas, quizá la producción más significativa será la de los cinco cuadros de Paul Cézzanne (1839-1906), Los jugadores de cartas (ca. 1895), donde aparece el enfrentamiento entre dos personalidades (Cámara y Carrió-Invernizzi, 2015; Carlo, 1998; Charles, Manca, McShane y Wigal, 2006; Cruzada, 1870; Frascina, Blake, Fer, Garb y Harrison, 1998; García, 1999; González Aja, 1988, 1990; Gracia, 1978; Mena, 2000; Riordan y Krüger, 2003; Zambrano, 2012).

Si centramos aún más el análisis de los grabados, concertándolo a los juegos de los niños, se podría analizar el momento infantil y pedagógico de la época, a partir de los cuadros. Como consecuencia, en lo que hace referencia a este marco de estudio, destacan diferentes artistas. A modo de ejemplo cabe citar a: Bartolomé Esteban Murillo (1617-1682) con Sagrada Familia del pajarito (1650), Niños jugando a dados (1665-75) o Invitación al juego de la argolla (1670); Jean Simón Chardin (1699-1779) con El castillo de naipes (1737), Niña con raqueta y volante (1737) o El niño de la peonza (1738). Pero una de las figuras que trató los juegos en la infancia de forma intensa fue Francisco de Goya (1746-1828), que pintó diversos cuadros relacionados con las diversiones y el esparcimiento de la juventud ${ }^{1}$. Así, de su extensa producción destacan las pinturas siguientes: La cometa (1778), Muchachos cogiendo fruta (1778), Niños inflando vejiga (1778), Niños del carretón (1779), Las gigantillas (17911792), La gallina ciega (1788), El pelele (1791-1792), El columpio (1779), Muchachos jugando a soldados (1779), El niño del árbol (1780), Muchachos trepando a un árbol (1791-

${ }^{1}$ El ocio y la diversión son elementos claves que trata Francisco de Goya, como es el caso de La merienda (1776), Baile a orillas del Manzanares (1776-1777), El paseo de Andalucía o La maja y los embozados (1777), La feria de Madrid (1779), El majo de la guitarra (1779), Niños con perros de presa (1786), La Pradera de San Isidro (1788), Los zancos (1791-1792), Los cómicos ambulantes (1793). Goya también trata todo un conjunto de obras relacionadas, por ejemplo, con la tauromaquia. Nota: Las obras se pueden visualizar en la página web del Museo del Prado: https://www.museodelprado.es/coleccion/artista/goya-y-lucientes-francisco-de/39568a1781b5-4d6f-84fa-12db60780812 
1792) y Pepito Costa y Bonells (1813) -con claros símbolos militares-. Y a estas producciones habría que añadir todo un conjunto de otros cuadros, grabados... que van apareciendo a lo largo de la historia, y en los cuales el elemento lúdico está presente (Carmona, 2009; Córdoba, Herrador, Martínez y Bueno, 2011; García y Llull, 2009; González Aja, 1992; Herrador, 2013; Mena, 2000; Mestre, 1973; Pelegrín, 1992).

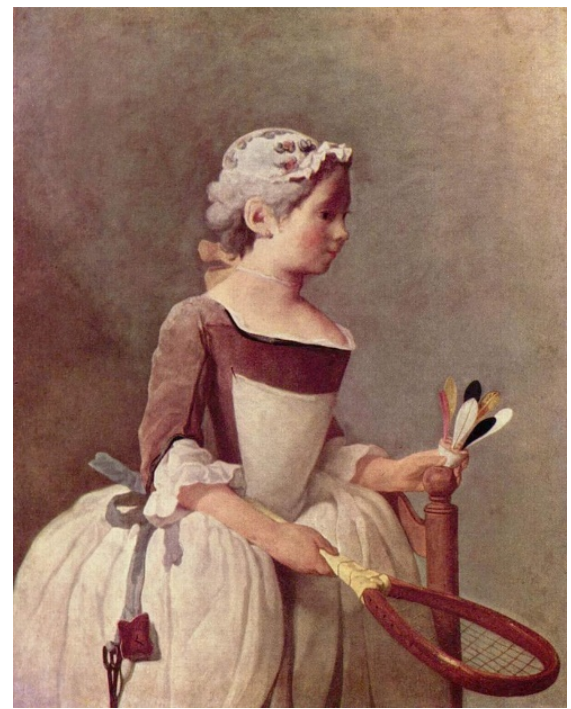

Imagen 1: Niña con raqueta y volante (1737), de Jean Simon Chardin ${ }^{2}$

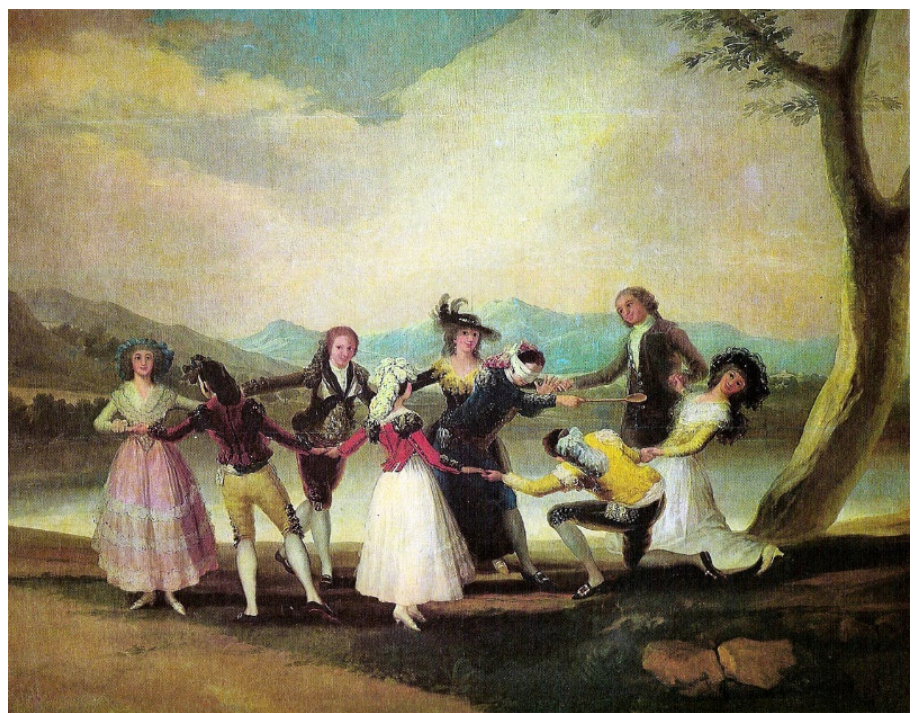

Imagen 2: La gallina ciega (1788), de Francisco de Goya ${ }^{3}$

Finalmente, en este tratamiento del juego y los niños, no hay que dejar de mencionar todo un conjunto de grabados lúdicos que son recopilatorios de cada época. Jacques Stella (1657, 1990) con sus Juegos y pasatiempos de la infancia 4 , o Joan Amades con sus aucas de juegos (Amades, 1947; Brasó y Collell, 2016; Brasó y Torrebadella, 2015) son dos figuras representantes de esta intencionalidad de describir y plasmar las prácticas de los niños. Juntamente con estos aspectos se encuentran cantidad de libros de juegos que entre sus páginas esconden grabados de los niños (Torrebadella, 2009, 2011), y que aunque no sean obras pictóricas -y por lo tanto se escapan del objeto de estudio de esta investigación-, su análisis tiene que permitir interpretar, entender y justificar la sociedad de cada época.

El objeto de estudio se centra en la obra pictórica de Peter Brueghel titulada Juegos de niños $(1560)^{5}$ dentro de la sociedad del s. XVI, en un momento en que el niño no era considerado como tal, sino como un hombre en potencia (Ariès, 1960). Concretamente el objetivo de la investigación pretende analizar la tipología de actividades en los Juegos de niños, relacionándolas con la praxiología motriz y con el contexto social de la época (Lavega y Navarro, 2014). De este modo se analiza la lógica interna ${ }^{6}$ de estas prácticas, su carácter motriz y el uso o no de algún instrumento. Asimismo, se ha estudiado el repertorio de cuadros

\footnotetext{
${ }^{2}$ Imagen extraída de: https://es.wikipedia.org/wiki/Muchacha_con_el_juego_de_la_pluma\#/media/File:JeanBaptiste_Sim\%C3\%A9on_Chardin_002.jpg ${ }^{3}$ Imagen extraída de:

https://es.wikipedia.org/wiki/La_gallina_ciega_(Goya)\#/media/File:La_gallina_ciega_(Goya).jpg ${ }^{4}$ Título original: Les jeux et plaisirs de l'enfance

${ }^{5}$ Nombre original Kinderspiele.Tomado de los datos en el Museu de Historia del Arte de Viena (Kunst Historisches Museum Wien). Disponible en:

http://www.khm.at/objektdb/detail/321/?offset=27\&lv=list\&cHash=d3b69e5402a4f3f06fa89ca8e997219e

${ }^{6} \mathrm{Se}$ entiende por lógica interna el "Sistema de los rasgos pertinentes de una situación motriz y de las consecuencias que entraña para la realización de la acción motriz correspondiente" (Parlebas, 2001, 302). El tratamiento de los juegos según este punto de vista permite describir las interacciones entre los participantes (Brasó y Torrebadella, 2015; Torrebadella y Brasó, 2014)
} 
Andújar, I., y Brasó, J. (2017). La lógica interna en Los Juegos de niños (1560) de Peter Brueghel. RICYDE. Revista internacional de ciencias del deporte, 50(13), 426-441.

del pintor, hecho que permite interpretar un cierto ideario, su modo de ver la vida y algunos aspectos del entorno social. La metodología se ha basado en el tratamiento de las principales fuentes documentales que relatan e interpretan la obra de Brueghel (Castelli, 2007; Herrador, 2005, 2012; Elschenbroich, 1979; McNeill, 2000; Navacerrada, 2007, 2012; Pelegrín, 1992, 1998), juntamente con el análisis taxonómico del conjunto de los juegos que aparecen en el grabado.

La justificación de un estudio que se fije en una imagen, un cuadro, un retrato... viene dada por el hecho de poder ser un elemento de construcción histórica (Grosvenor, 2007, 2016). Tiene además el valor añadido que puede dar información sobre aspectos que los textos pasan por alto. De todas formas, hay que ser consciente, como Ariès (1960) remarca, que las imágenes no son el reflejo de la realidad, sino que se convierten en la idea de una sociedad para los creadores de estos cuadros, fotografías, retratos que quieren representar un momento, una época, un lugar... En esta línea, Pérez Vejo ya comenta que:

\begin{abstract}
"si queremos, que queremos saber cómo una sociedad "era" es muy posible que el vestigio más apropiado no sean las imágenes sino otro tipo de documentos; pero si queremos saber cómo una sociedad se veía a sí misma y al mundo que la rodeaba, la trama en que articulaba sus creencias colectivas, las mentalidades que permitían funcionar y legitimar determinadas estructuras sociales y políticas, las identidades colectivas que hacían a los individuos sentirse miembros de una comunidad política o social $y$, en definitiva, el cúmulo de ideas preconcebidas y prejuicios morales a partir de los que toda sociedad se articula, las imágenes se convierten en un vestigio imprescindible" (Pérez, 2012, 29)
\end{abstract}

Añade que quizá el objetivo de los historiadores tiene que ir en la línea de "no contar cómo fueron las sociedades del pasado, sino cómo se imaginaron que eran. Objetivo para el que las fuentes icónicas no solo son necesarias sino imprescindibles" Pérez $(2012,29)$. Seguidamente se aporta información, antes de afrontar el objeto de estudio, de Brueghel, autor de Juegos de niños, para poder comprender el sentido, motivo y filosofía de su obra pictórica.

Peter Brueghel (c. 1525-1569)

Peter Brueghel "el Viejo", nació supuestamente en Breda (Holanda) y murió en Bruselas (Bélgica). Fue un artista flamenco de la pintura, discípulo de un "romanista" (Bennassar, Jacquart, Lebrun, Denis y Blayau, 2005). En 1551 su nombre aparece ya en el registro del gremio de pintores de San Lucas de Amberes, y es considerado un maestro. Viaja por Francia, Italia y Suiza y su fuente de inspiración se encuentra en las vistas a los Alpes y a los Apeninos. Estuvo en Italia dos años y ya pintó cerca de Roma su Paisaje fluvial con dibujante (1553), que como novedad trata el género paisajístico, que aún no se había institucionalizado. La pintura muestra así un nuevo punto de vista, donde el paisajista se integra en el arte, y donde aparece una nueva visibilidad de la Tierra, un nuevo pensamiento, una nueva mirada del mundo. Después de volver de Italia -lugar donde se inicia y desarrolla el Renacimiento a partir del siglo XV-, en 1555 se instaló en Amberes, trabajando para el impresor y grabador Hieronymus Cock. Fue entonces cuando su nombre cogió fama gracias a un conjunto de grabados satíricos, moralizadores y con similitudes a la obra de el Bosco. La publicación de Los siete pecados capitales (c. 1557) confirmó la reputación de Brueghel como grabador. En 1562 se instaló en Bruselas, donde se casó y tuvo dos hijos, en 1564 y 1568, también pintores: Pieter Brueghel "el Joven", y Jan Brueghel "el Viejo". De todas formas, no fue hasta principios del s. XX que los críticos empezaron a valorarlo como la figura relevante que fue (Balius, 2008; Soto, 2010).

\footnotetext{
${ }^{7}$ También se le encuentra nombrado como Pieter. A la vez se encuentra el apellido 'Bruegel' y Brueguel. Sus hijos, Peter Brueghel, el joven, y Jan Brueghel, devolvieron la "h" al apellido que el padre había eliminado. En este artículo se citará como Peter Brueghel.
} 
Sus escenas sobre paisajes, representaciones de la realidad de la vida campesina, el folclore, el ocio o los juegos (Prohaska, 2004), además de su tratamiento innovador de temática religiosa -donde los acontecimientos bíblicos aparecen en escenarios panorámicos y vistos desde arriba-, juntamente con el simbolismo que incluye significó una alta valoración del artista. Este hecho no quita que la mayoría de sus obras fuesen encargos. Han perdurado unas 40 pinturas. La actitud humanista y filosófica hacen que su figura sea considerada del Renacimiento, aunque, eso sí, sin influencia italiana (Bennassar y col., 2005; Departamento de Educación, 2005; López, 2013; Riaño, 2013; Saccà, 2011).

Brueghel realizó obras que aún gozan de popularidad, como el Combate entre don carnaval y doña cuaresma (1559) o los Proverbios flamencos (1559). Pero en el caso que nos ocupa y antes de entrar en la obra de los Juegos de niños, habría que fijarse en Cazadores en la nieve (1565), donde el pintor incluye el patinaje sobre hielo, el hoquei, el curling, la peonza, la lucha, la danza y otros juegos de niños y adultos en el hielo. Es curioso destacar el parecido que tendrá posteriormente otro cuadro de su hijo Brueghel "el Joven", con el de su padre. Nos referimos a Paisaje nevado (1601). En este también se describirán en el hielo diferentes juegos.

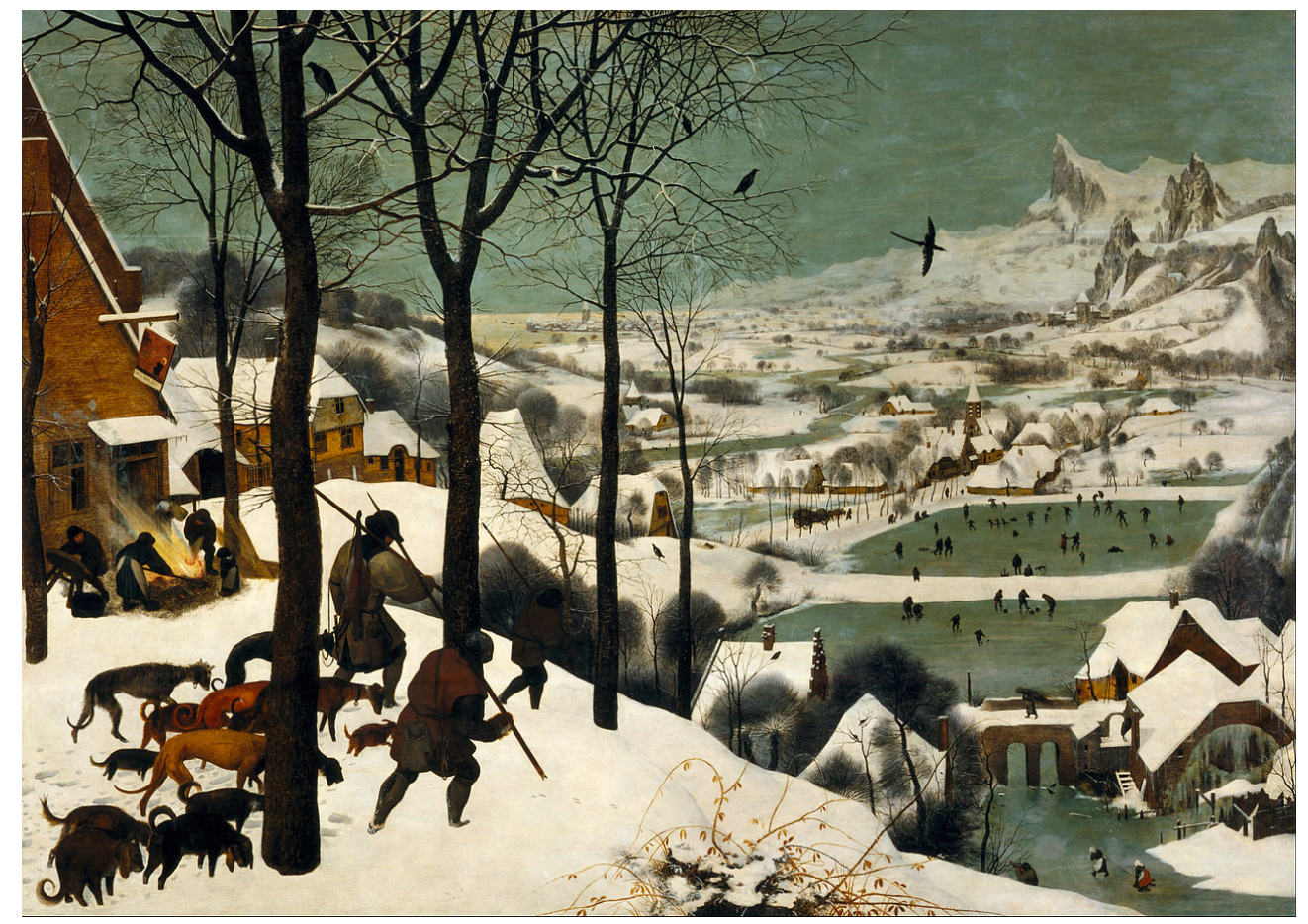

Imagen 3: Grabado de los Cazadores en la nieve (1565), en este caso de Peter Brueghel, el Joven $^{8}$

Asimismo, y volviendo a Brueghel "el Viejo", también en el Censo en Belén (1566) se encuentran juegos como la peonza, jugar a empujarse encima de una madera o jugar a trineos. También habría que destacar pinturas en las que aparecen otras prácticas corporales como son las danzas. Estas son: El baile nupcial (1566) y la Danza de los campesinos (1568). Queda claro, por lo tanto, la motivación del pintor por retratar elementos de la vida cotidiana, de las costumbres, del folclore.

\footnotetext{
${ }^{8}$ Disponible en:

https://commons.wikimedia.org/wiki/File:Pieter_Bruegel_the_Elder_-_Hunters_in_the_Snow_(Winter)__Google_Art_Project.jpg\#
} 
El tema de estudio de los Juegos de niños (1560) destaca por "su carácter hondamente narrativo, como de estampa, pero sobre todo también a su tema: la representación de una diversión aparentemente intemporal" (Elschenbroich, 1979, 87). Así, el ocio, diversión, libertad y triunfo de la vida que aparece en el cuadro tiene su opuesto en El Triunfo de la Muerte (1562-1563), obra del mismo pintor. De todas formas, esta dicotomía se escapa del objetivo del estudio, aunque tiene que ser motivo de reflexión.

\section{Los Juegos de niños (1560)}

La obra Juegos de niños -óleo sobre tabla de 118x161cm.- se encuentra en el Museo de Historia del Arte de Viena (García, 1999; Prokaska, 2004) -Kunsthistorisches Museum-. El pintor marcó la línea del horizonte en el grabado, en un punto de vista muy alto -como también hizo con el Retorno de los rebaños (1565)-. Por este motivo el observador del cuadro tiene la sensación de visualizar toda la actividad que sucede en el grabado como si estuviese volando.

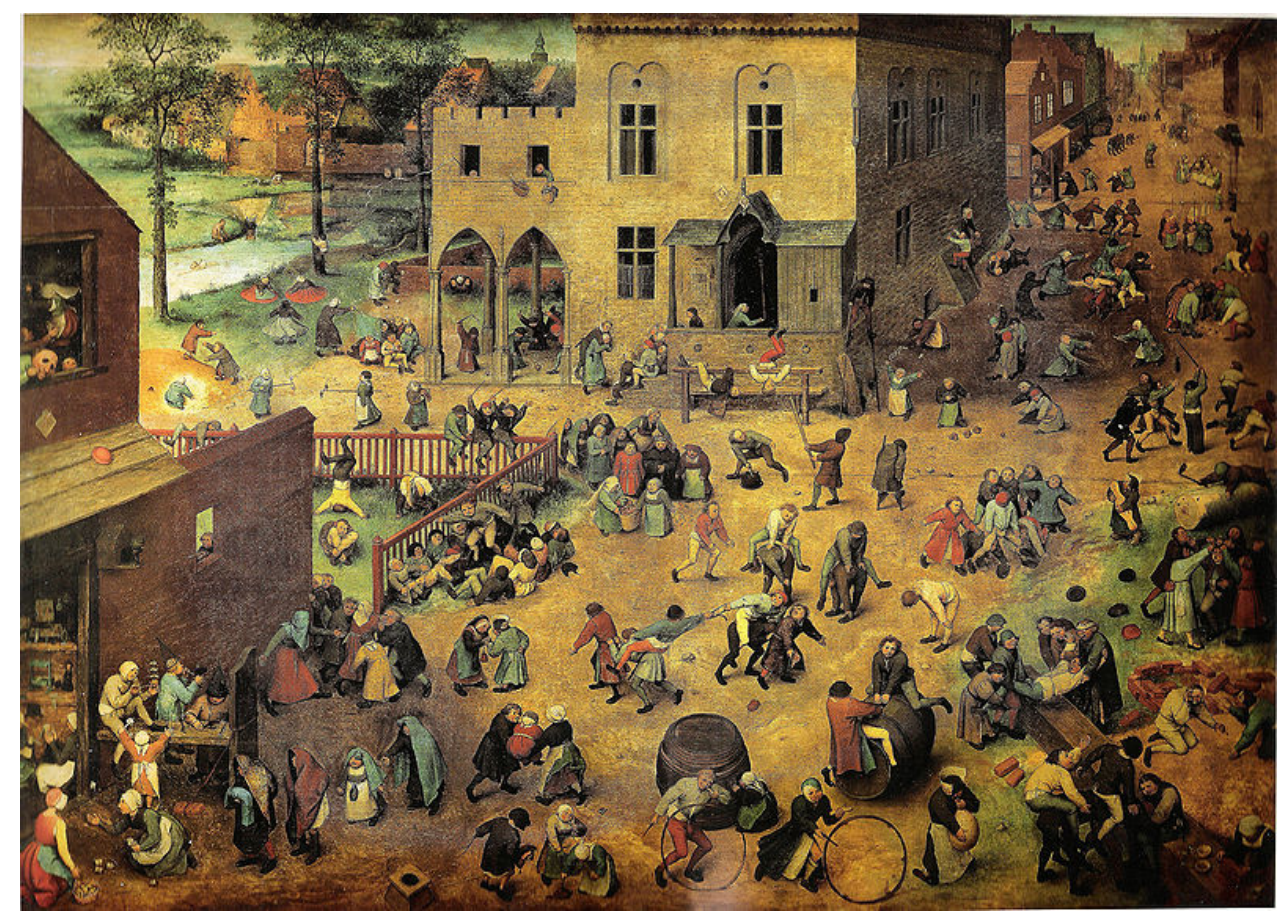

Imagen 4: Grabado de los Juegos de niños (1560), de Peter Brueghel "el Viejo"9

Su simbolismo, relación e importancia de los juegos con la sociedad del s. XVI, lo hacen digno de análisis. De este modo, como en otros cuadros del mismo autor, se intuye una voluntad de crítica y liberación del modelo social en los Países Bajos de Felipe II (Valladolid, 1527-El Escorial, 1598). Hay que decir que, aunque Felipe II valoró la cultura y la sociedad de los Países Bajos, sus intentos de control por mantener la unidad política y religiosa, desencadenaron en fuertes movimientos protestantes, y en una rebelión. A estos sucesos se le sumaron otros y le siguió la guerra entre Francia y España. El Tratado de Vervins en 1598, significó el fin a la guerra entre estos dos países y la cesión de los Países Bajos al archiduque Alberto de Austria y a la princesa Isabel Clara Eugenia.

Por lo tanto, desde el punto de vista de Brueghel, la obra permite intuir la situación políticasocial de la época. El paisajista propone un ideal democrático, una República -como se simboliza en el grabado, con numerosos niños que se autogestionan, sin controles ni disputas- (Elschenbroich, 1979). A la vez, el lugar de la práctica, alejado del centro de la

\footnotetext{
${ }^{9}$ Disponible en: https://es.wikipedia.org/wiki/Archivo:Children.jpg
} 
ciudad, incluso fuera de esta (a extramuros) indicaría la voluntad de Brueghel por representar la clase joven y baja de la población, la cual no tiene acceso a ningún tipo de educación pedagógico-formal. La única formación que tiene es la del trabajo para cubrir las necesidades básicas. Quizá por ello las expresiones faciales de los participantes en la obra pictórica son mayoritariamente serias, a modo de adulto preocupado. Incluso los instrumentos lúdicos son, como interpreta Elschenbroich (1979), reciclajes de material del mundo de los mayores, o instrumentos autoconstruidos por los niños. Asimismo, este hecho justificaría el interpretar la obra como el caos en un día festivo, donde los niños pueden liberarse de la fatigosa rudeza de los trabajos y entretenerse, como la clase alta, disfrutando de un pequeño momento de ocio.

La pintura detalla muchos juegos de niños al aire libre en verano, en Flandes, y representa una pequeña enciclopedia de juegos (Pelegrín, 1998). Y lo hace de un modo sencillo, con una actitud por retratar aspectos habituales, espontáneos y cotidianos del folclore (Castelli, 2007; Herrador, 2005, 2012; McNeill, 2000). La espontaneidad y cotidianidad intemporal de las prácticas lúdicas se hace evidente al visualizar la obra. También permite analizar el estatus que tenía el niño, el cual era considerado como un adulto en miniatura. La vestimenta y la propia actitud, seria y a menudo de sufrimiento muestran esta diferencia entre la juventud del s. XVI y la actual (Elschenbroich, 1979; Herrador, 2013; Menzel, 1966; Pavón, Herrador, Sánchez y Márquez, 2011; Varela, 1986).

Donata Elschenbroich (1979) detalla, asimismo, que aparecen los chiquillos como una especie: con sus cuerpos desproporcionados. Además, hay dificultad a menudo por diferenciar el sexo del niño/a representado -a causa de la vestimenta, que no permite esta distinción. A la vez, tampoco es posible determinar exactamente el nivel social, aunque la vestimenta no es en ningún caso representativa de la clase alta de la sociedad-. De este modo:

\begin{abstract}
"Los niños parecen tener cuerpos más cortos, aún más rechonchos y menos individualizados, o sea aparecen como una especie. Brueghel no exhibe a los niños como seres juguetones y graciosos, sino que, al contrario, lo grosero y rechoncho de sus proporciones está incluso exagerado mediante su pequeño tamaño. Cráneos redondos que ocultan el cuello, hombros anchos y redondos, espaldas en parte casi jorobadas, fuertes piernas y pesados, enormes zapatos (¿zapatos de adultos?). Los rostros no están individualizados y son gruesos y anchos, la expresión reside sobre todo en los movimientos y en la relación de las figuras entre sí. Los cabellos cortados sin ninguna forma y greñudos, muchas veces una capucha enormemente grande cubre la mitad de la cara (un motivo predilecto en Brueghel...). Ninguna vestimenta infantil especial... de tal forma que no siempre es posible decidir si se trata de vestidos de niño o de niña" (Elcshenbroich, 1979, 89).
\end{abstract}

Asimismo, esta idea del niño como un adulto se comprueba en el material utilizado para el propio juego. Siguiendo el análisis detallado de Elschenbroich apreciamos que se ven pocos juguetes. Lo que se comprueba es la utilización de material, instrumentos, aparatos... pertenecientes al mundo del trabajo o vida del adulto (sombrero, escoba, banco, vestidos, flores, ladrillos, flauta, tambor...). Algunos de estos son:

"El barril, el aro fabricado a partir de él, cestas, escoba, cercado, la barra para uncir los
caballos, la vejiga de cerdo. Los objetos que pueden ser llamados juguetes en sentido estricto,
han sido fabricados posiblemente por los niños mismos: remolino de viento, yoyo, zancos,
máscaras, látigo, balanza, huesitos para jugar a los dados. Únicamente el caballo de palo y
posiblemente las muñecas son juguetes producidos por los adultos expresamente para los
niños" (Elcschenbroich,1979, 89).

En esta línea es destacable que el mundo de los adultos está sólo representado por estos elementos y por los edificios, siendo la aparición de personas mayores, insignificante -solo dos en todo el cuadro-.

Además, este uso del material, y la facilidad por la fabricación de los instrumentos para el juego, por parte del niño, indican que el factor de posesión y competencia, en el s. XVI, aún no está arraigado -además de interpretarse que este uso del material no tiene prohibiciones, ni 
Andújar, I., y Brasó, J. (2017). La lógica interna en Los Juegos de niños (1560) de Peter Brueghel. RICYDE. Revista internacional de ciencias del deporte, 50(13), 426-441.

limitaciones por sus caracteres de peligrosidad-. Como consecuencia, el aprender a compartir, es decir reprimir la posesión individual, aún no se ha convertido en "uno de los objetivos pedagógicos del comportamiento del niño" (Elschenbroich, 1979, 90). Por lo tanto, no hace falta el control del adulto, ya que no hay disputas entre chiquillos por el material. Por otro lado, la autora, también centra la atención en el tipo de prácticas, muchas de ellas físicas, jugadas en la sociedad también por los mayores y que permiten a la vez entrever la aparición de reglas, normas y modos de operar del mundo adulto. A modo de ejemplo, aunque las actividades parecen no tener ni sexo ni edad para los practicantes, en algunas se visualiza el control-dirección del juego por parte de un niño más grande. En lo que se refiere al sexo de los practicantes, como en otros juegos tradicionales de otras épocas y lugares, son variables (Brasó y Torrebadella, 2015). Siguiendo a Elschenbroich, se evidencia que Brueghel solo representa juegos de niños, hecho que hace también entrever la progresiva importancia que se le empezaba a dar al juego infantil.

Pelegrín $(1992,369)$ comenta que la obra de Brueghel "es un valioso testimonio de los juegos infantiles del siglo XVI, guiando la comprensión al visualizar la acción y la gestualidad" corporal. En la misma línea, Navacerrada $(2007,2012)$ también afirma que los juegos pintados en el cuadro tienen gran valor para estudiar los juegos de la Edad Moderna juntamente con los grabados de Jacques Stella $(1657,1990)-$. Visto de este modo el cuadro se convierte en una significativa enciclopedia de juegos (Herrador, Zagalaz, Martínez, Rodríguez, 2006)

La identificación de las prácticas que aparecen en el grabado no es tarea sencilla. Diferentes estudiosos han tratado este aspecto, como Hills (1940, 1956), Portmann (1961), Elschenbroich (1979), Vanden (1982); Pelegrín (1998), Navacerrada (2007, 2010, 2012) ${ }^{10} \mathrm{o}$ Breyer (2010). Para el estudio, se ha tomado la clasificación de Navacerrada, que es la más actualizada y la que detalla más juegos, concretamente 87 [tabla 1].

Tabla 1: Nombre de cada juego -adaptado de Hills (1956), Navacerrada (2007), Pelegrín (1998) -

\begin{tabular}{|c|l|}
\hline $\mathbf{N}$ & \multicolumn{1}{|c|}{ Juego } \\
\hline 1 & Las tablas \\
\hline 2 & Jugar a las muñecas \\
\hline 3 & Representar una misa \\
\hline 4 & La bramadera \\
\hline 5 & Hacer pompas de jabón \\
\hline 6 & $\begin{array}{l}\text { Jugar con un sombrero } \\
\text { de paja }\end{array}$ \\
\hline 7 & Cazar pájaros \\
\hline 8 & Jugar a atacar tu mascota \\
\hline 9 & El sonajero \\
\hline 10 & Representar un bautizo \\
\hline 11 & Jugar a caballeros \\
\hline 12 & $\begin{array}{l}\text { Tocar la flauta y el } \\
\text { tambor }\end{array}$ \\
\hline 13 & Candela, papasal \\
\hline 14 & $\begin{array}{l}\text { Jugar a la sillita de la } \\
\text { reina }\end{array}$ \\
\hline 15 & Esconderse en un barril \\
\hline
\end{tabular}

\begin{tabular}{|c|l|}
\hline $\mathbf{N}$ & \multicolumn{1}{|c|}{ Juego } \\
\hline 30 & Pares o nones \\
\hline 31 & Salto de palomita \\
\hline 32 & Lucha de caballos \\
\hline 33 & Pídola \\
\hline 34 & Bajo pierna \\
\hline 35 & Mona de pascua \\
\hline 36 & Gato repelado \\
\hline 37 & Abejorros \\
\hline 38 & La Máscara \\
\hline 39 & El columpio \\
\hline 40 & Juegos en la arena \\
\hline 41 & El rey de la montaña \\
\hline 42 & Saltar la valla \\
\hline 43 & El molinete \\
\hline 44 & La carraca \\
\hline
\end{tabular}

\begin{tabular}{|c|l|}
\hline $\mathbf{N}$ & \multicolumn{1}{|c|}{ Juego } \\
\hline 59 & Bailar la peonza \\
\hline 60 & Golpear la peonza \\
\hline 61 & Volar la cinta \\
\hline 62 & $\begin{array}{l}\text { Colgar una panera en la } \\
\text { ventana }\end{array}$ \\
\hline 63 & Trae ese costal \\
\hline 64 & La vara en equilibrio \\
\hline 65 & Colgando de la barra fija \\
\hline 66 & Dar vueltas en la barra \\
\hline 67 & Zancos grandes \\
\hline 68 & $\begin{array}{l}\text { El pajarillo huidizo que } \\
\text { se escapa }\end{array}$ \\
\hline 69 & Castilletes \\
\hline 70 & Los bolos \\
\hline 71 & Gurria, pina \\
\hline 72 & Hoyuelos \\
\hline 73 & Trepar por el muro \\
\hline
\end{tabular}

${ }^{10}$ En la página web de la revista digital Balises de la Biblioteca Pública de Francia, se pueden consultar de modo interactivo los juegos y su nombre. Disponible en: http://balises.bpi.fr/education/jeux-denfants-de-pieterbruegel-lancien. 
Andújar, I., y Brasó, J. (2017). La lógica interna en Los Juegos de niños (1560) de Peter Brueghel. RICYDE. Revista internacional de ciencias del deporte, 50(13), 426-441.

\begin{tabular}{|l|l|}
\hline 16 & Dos, subidos en un tonel \\
\hline 17 & Jugar a los aros \\
\hline 18 & Inflar un globo \\
\hline 19 & Masculillo \\
\hline 20 & Churro \\
\hline 21 & El robaterrenos \\
\hline 22 & Juegos de construcción \\
\hline 23 & Jugar a las tiendas \\
\hline 24 & Pistolas de agua \\
\hline 25 & $\begin{array}{l}\text { Ponerle una casita a un } \\
\text { pájaro }\end{array}$ \\
\hline 26 & La gallinita ciega \\
\hline 27 & Dar volteretas \\
\hline 28 & Hacer el pino \\
\hline 29 & Hacerse una bola, nudo \\
\hline
\end{tabular}

\begin{tabular}{|l|l|}
45 & $\begin{array}{l}\text { Montar a caballo en una } \\
\text { valla }\end{array}$ \\
\hline 46 & Representar una boda \\
\hline 47 & La piñata \\
\hline 48 & Los zancos \\
\hline 49 & El highí: este peral \\
\hline 50 & Nadar con flotador \\
\hline 51 & $\begin{array}{l}\text { Nadar hasta las } \\
\text { pantorrillas }\end{array}$ \\
\hline 52 & Nadar y guardar la ropa \\
\hline 53 & $\begin{array}{l}\text { Representar una corona } \\
\text { de flores }\end{array}$ \\
\hline 54 & Ondular los vestidos \\
\hline 55 & Trepar a un árbol \\
\hline 56 & Juego con bolas, petanca \\
\hline 57 & La viejecita \\
\hline 58 & Hacer pipí \\
\hline
\end{tabular}

\begin{tabular}{|r|l|}
\hline 74 & ¡A luchar! \\
\hline 75 & Olla de miel \\
\hline 76 & La cola del diablo \\
\hline 77 & Las procesiones \\
\hline 78 & Los tazos; El palmo \\
\hline 79 & Bailar el sombrero \\
\hline 80 & Pilla-pilla \\
\hline 81 & El portero de la casa \\
\hline 82 & $\begin{array}{l}\text { Petición de boda o los } \\
\text { oficios }\end{array}$ \\
\hline 83 & Montar a caballito \\
\hline 84 & Parir la gata \\
\hline 85 & El caballo sinuoso \\
\hline 86 & Los oficios \\
\hline 87 & $\begin{array}{l}\text { La fogata; la hoguera de } \\
\text { San Juan }\end{array}$ \\
\hline
\end{tabular}

\section{Análisis de la lógica interna de los Juegos de niños}

Estos juegos quedan bien plasmados en diferentes propuestas educativas en el aula. Es la materia de educación física la que tiene una mayor significación para tratarla. Las iniciativas en este sentido son diversas. Destaca la actividad cooperativa e interdisciplinar de Pubill (2009), analizando el cuadro, investigando, haciendo representaciones por parte de los alumnos, lo que da muestras de incidir en un mejor desarrollo integral a partir de la obra pictórica. Asimismo, aparecen otras propuestas como la de la Universidad Pablo de Olavide ${ }^{11}$ (2013), la de Manzano (2014) $)^{12}$, la de Díaz y Mango (2011) ${ }^{13}$, la de Herrador (2005), la del Instituto Ferran Casablancas ${ }^{14}$ (Pérez y Benet, 2014), o las citadas por Balius (2008), en la que el juego aparece de forma más escénica en la educación.

Por lo tanto, es posible y pertinente un tratamiento a fondo de los Juegos de niños, en el ámbito motriz, es decir, en su lógica interna. De este modo, se favorecerá a la recuperación y tratamiento histórico-social -en este caso del juego y de la infancia-. A la vez este análisis del cuadro puede facilitar la tarea a cualquier docente en educación física que quiera incorporar los juegos de Brueghel en sus sesiones y en la programación anual.

Se pretende así recuperar la tradición a partir de los cuadros y la posibilidad de realizar una intervención didáctica en el aula, en una idea parecida a la de Carmona (2009). Como consecuencia, la propuesta que a continuación se ofrece, se basa en un análisis sobre la lógica interna (Parlebas, 2001) de los juegos aparecidos en el grabado. De este modo, partimos de investigaciones que analizan la motricidad en la obra de Brueghel, como la de Calmels (2007), la de Navacerrada (2007), que juntamente con la de Pubill (2009) o Hills (1940) hacen un estudio detallado del cuadro.

El análisis de la lógica interna tiene su utilidad en la medida que permite analizar el tipo de juego practicado por la sociedad y hacernos una idea de las características comunes de éstos $\mathrm{y}$, por lo tanto, indagar en el modo de hacer social. A la vez, su clasificación tiene que servir

\footnotetext{
${ }^{11}$ Disponible en: https://youtu.be/rCuUOGvAHsM

12 Disponible en: http://webquestpieterbruegel.weebly.com/

${ }^{13}$ Disponible en: http://uruguayeduca.edu.uy/Portal.Base/Web/VerContenido.aspx?ID=210181

${ }^{14}$ Disponible en: https://youtu.be/pxkMRL0hASk
} 
Andújar, I., y Brasó, J. (2017). La lógica interna en Los Juegos de niños (1560) de Peter Brueghel. RICYDE. Revista internacional de ciencias del deporte, 50(13), 426-441.

para organizar estas actividades en el ámbito motriz, específicamente en la materia de Educación Física. De este modo se analizan la tipología de estos juegos tradicionales según su motricidad, clasificando las prácticas motrices como aquellas en las que aparece de manera significativa alguna cualidad física básica, y las no motrices, como aquellas en las que estas cualidades tienen una baja aparición, ya sea por su carácter sedentario o por su sentido simbólico. Las diferencias interpretativas sobre la motricidad de los juegos por parte de los diferentes autores hacen que los resultados estadísticos, pudiesen variar, de una interpretación a otra, aunque, por lo que se ha comprobado, no significativamente, en torno a un $5 \%$. Se ha optado por detallar esta división con el sentido de poder analizar el carácter motriz en los Juegos de niños. Además, de todos los juegos - motrices y no motrices- se analiza su lógica interna, relacionada con los compañeros, con los adversarios, y también en función del uso o no de material ${ }^{15}$, en una línea análoga a propuestas que analizan aspectos similares y ya publicadas (Brasó y Collell, 2016; Brasó y Torrebadella, 2015; Lagardera y Lavega, 2003; Parlebas, 2001, 2003).

Los 87 juegos que aparecen en el cuadro se han analizado por lo que respecta a la lógica interna. Asimismo, también es básico analizar el carácter infantil y masculino que se entrevé al mirar el grabado y que Navacerrada $(2007,2012)$ y Pelegrín $(1992,1998)$ estudian. Hacen constar estos autores que, de los 247 personajes representados, éstos se dividen en 168 niños, 78 niñas y un adulto ${ }^{16}$. El tratamiento extenso y profundo que proporciona Pelegrín (1992, 1998) juntamente con los trabajos de Navacerrada (2007, 2012), son los que se han utilizado como base para el estudio, aunque también se han tenido en cuenta las propuestas ya citadas, para dar más validez y verificar los resultados (Breyer, 2000; Hills, 1940, 1956; Pavón y col., 2011, Mehl, 1990; Turcot, 2016; Vanden, 1982).

A partir de todas estas informaciones se ha elaborado un análisis estadístico, procedimiento que tiene que permitir interpretar modelos sociales de la época.

\section{Resultados}

De los datos anteriores se comprueba que en el grabado de los Juegos de los niños hay un $68 \%$ de niños y sólo el 32\% son niñas ${ }^{17}$ (Navacerrada, 2007, 2012; Pelegrín, 1992, 1998).

Por lo que hace referencia al carácter motriz y no motriz, de los juegos, su relación con la lógica interna y con el uso de materiales para la práctica, los datos obtenidos del análisis estadístico se resumen en las tablas 2 y 3 .

Tabla 2: Relación entre la tipología de juegos según su motricidad, y su lógica interna analizada

\begin{tabular}{|l|c|c|c|}
\cline { 2 - 4 } \multicolumn{1}{c|}{} & Juegos motrices & Juegos no motrices & Total \\
\hline Sólo compañeros & $23 \%$ & $21 \%$ & $44 \%$ \\
\hline Sólo adversarios & $7 \%$ & $2 \%$ & $9 \%$ \\
\hline Con compañeros y adversarios & $2 \%$ & $1 \%$ & $3 \%$ \\
\hline Sin compañeros ni Adversarios & $20 \%$ & $24 \%$ & $44 \%$ \\
\hline Total & $52 \%$ & $48 \%$ & $100 \%$ \\
\hline
\end{tabular}

\footnotetext{
${ }^{15}$ Se ha optado por interpretar como material cualquier instrumento u objeto utilizado para la práctica lúdica, aunque sea del mundo del adulto, en la línea de las ideas de Elschenbroich (1979).

${ }^{16}$ Elschenbroich detalla que aparecen dos adultos.

${ }^{17}$ Para los cálculos estadísticos se han omitido los decimales, los cuales no son significativos en estudios de esta tipología.
} 
Se comprueba una aparición similar de prácticas motrices (52\%) y no motrices (48\%) en la obra de Brueghel. Además, la lógica interna, en la línea de la clasificación de Parlebas (2001) es parecida en los dos grupos de juegos, siendo aquellos en los que el jugador actúa sólo con compañeros (44\%), y los que actúa individualmente (44\%), los que tienen una aparición predominante en el cuadro [tabla 2]. Por lo tanto, es destacable la principal predominancia de juegos donde no aparecen adversarios. Agrupando así la totalidad de estas prácticas sin una oposición de un rival -tanto motrices (43\%), como no motrices (45\%)-, el 88\% de los juegos son de esta tipología. Como consecuencia, los juegos de oposición, ya sean con compañeros o no, solo obtienen un $12 \%$ de aparición.

Tabla 3: Porcentaje de juegos atendiendo a su carácter motriz / no motriz y relacionándolo con el uso o no de material

\begin{tabular}{|l|c|c|c|}
\cline { 2 - 4 } \multicolumn{1}{c|}{} & Juegos motores & Juegos no motores & Total \\
\hline Con material & $33 \%$ & $37 \%$ & $70 \%$ \\
\hline Sin material & $19 \%$ & $11 \%$ & $30 \%$ \\
\hline Total & $52 \%$ & $48 \%$ & $100 \%$ \\
\hline
\end{tabular}

Por lo que respecta al uso o no de material, los resultados son bastante similares tanto si se trata de juegos motrices como de prácticas no motrices [tabla 3]. El uso de algún instrumento aparece en el 70\% de los juegos, y solo el 30\% de los juegos son sin material.

Como consecuencia de todo lo dicho, comparando las tablas 2 y 3 , y después de hacer el análisis estadístico pertinente, se obtiene que un $60 \%$ de los juegos cumplen la premisa de ser prácticas -motrices o no motrices-, sin adversarios -tanto si el sujeto actúa de forma individual como si lo hace con cooperación-, $\mathrm{y}$, a la vez, con algún uso de un instrumento.

\section{Discusión}

De todos estos resultados se infieren diferentes aspectos. Primeramente, de los datos estadísticos, se comprueba un equilibrio entre prácticas motrices y no motrices, lo que muestra la diversidad de juegos de niños en la sociedad de la época. También se comprueba que la lógica interna está bien alejada de la oposición-enfrentamiento entre jugadores. Así, los resultados obtenidos a partir del grabado están en acorde con la línea interpretativa de Elschenbroich (1979) relacionada con la baja competencia entre niños, gracias al exceso de material y la no necesidad de enfrentarse -hecho que no quiere decir que no se encuentren algunos juegos de enfrentamiento-.

Además, se infiere el carácter de no control de las prácticas por parte del adulto, lo que implica practicar juegos más o menos de una lógica interna sencilla y con una simplicidad en el reglamento. Este hecho no implica que no estén controlados. Ya se ha hecho mención del control a menudo de los propios niños. De este modo, la libertad lúdica de los niños visualizada en la obra de Brueghel también en la no aparición de adultos en el grabadoimplicaba estos tipos simples de práctica. Todo ello queda más validado todavía analizando la poca incidencia de juegos cuya tipología es de la mayor complejidad motora, cognitiva y, principalmente organizativa -los juegos sociomotrices de cooperación-oposición (Parlebas, 2001)-.

Por lo que respecta al uso de material, se comprueba la mayor predominancia de una herramienta, objeto... en los juegos con baja o nula incidencia motora -hecho obvio ya que estos juegos, en la mayoría de casos tienen por objetivo el dominio técnico de estos materiales-. En cambio, los juegos motrices, que implican el desarrollo de alguna cualidad 
Andújar, I., y Brasó, J. (2017). La lógica interna en Los Juegos de niños (1560) de Peter Brueghel. RICYDE. Revista internacional de ciencias del deporte, 50(13), 426-441.

física, el trabajo de esta cualidad es el propio objetivo de la práctica, motivo por el cual, en muchas actividades de este tipo, no hace falta utilizar ningún instrumento.

Los resultados estadísticos se relacionan, por lo tanto, con las aportaciones que tratan el no control del juego del niño -en la sociedad del s. XVI-. Como consecuencia se hace evidente la libertad y autonomía que los más pequeños tenían, en relación con la sociedad actual (Elschenbroich, 1979, Torrebadella, 2016; Varela y Álvarez-Uría, 1991). Todo ello se plasma en la alta cantidad de juegos con un bajo componente organizativo, hecho que implica que sean de práctica individual o como mucho con la cooperación de algún compañero.

Es destacable, pero, que muchos de estos juegos entraron posteriormente, durante los siglos XVII y XVIII en un proceso de pedagogización, en el que el Estado intentó organizar, guiar, orientar, adoctrinar... la población y los niños. La escuela, por lo tanto, como aparato ideológico en que se convirtió -en palabras de Althusser (1974, 1989)- buscó a partir de diferentes instrumentos el dominio de una clase sobre otra, y el juego fue una útil herramienta para este objetivo. Así, la idoneidad de unas u otras prácticas quedaba poco a poco regulada según el lugar y la época (García, 1999; González, 1993; Strosetzki, 1996).

\section{Conclusión}

El análisis de obras pictóricas y grabados como los de Brueghel tienen que ayudar a completar el entendimiento de la sociedad de una época, tratando de detallar así sus prácticas y lugares destinados a ello, sus ritos, sus vestimentas, el género de los participantes. El proceso civilizador de la sociedad iniciado en el s. XVI (Elias y Dunning, 1992) queda bien plasmado en el grabado, donde la mayoría de prácticas no son de enfrentamiento. Por lo tanto, la idea de pacificación y sensibilización son evidentes.

Además, la obra tiene que ayudar a comprender e inferir otras ideas relacionadas con la situación del niño en relación con el adulto, con la sociedad, o simplemente con las interrelaciones entre individuos. A modo de ejemplo, la obra pictórica, con dos tercios de participantes masculinos, da a entender el carácter masculino de la época. A la vez, destacan los pocos objetos del mundo de los niños, es decir, los juguetes propiamente dichos, lo que muestra una no importancia ni sensibilidad por la educación doméstica o privada de la infancia - la mayoría de instrumentos para el juego son del mundo del adulto o de fácil autoconstrucción para el niño-.

En este análisis social el no control por parte del adulto es más que evidente y permite intuir el poco interés por las primeras edades de la vida. De todas formas, la sensibilidad de Peter Brueghel por los juegos en los niños podría simbolizar un punto de inicio de la atención a los infantes $\mathrm{y}$, como consecuencia, lo que será el proceso de pedagogización lúdica, y el elemento clave en el que el juego se transformará.

En el caso que nos ocupa, el estudio ha permitido corroborar modelos en relación con el juego y la función social del niño en la sociedad de la época y a la vez, dibujar sus relaciones con los otros niños y el material utilizado para el juego. Estos últimos hechos están en la línea de los principales tratadistas. Las prácticas con una baja lógica motriz son, por lo tanto, las predominantes, lo que se relaciona con la ya citada no intervención del adulto en el juego de los niños y, a la vez, con el juego libre. Este hecho se contrapone, por ejemplo, con la tipología de juegos que aparecen hoy en la infancia, muchos de los cuales tienen una compleja lógica motriz y también un alto control y seguimiento de uno o más adultos.

Somos conscientes del hecho que el estudio es sólo es una muestra, a partir de un punto de vista concreto. Habrá que tratar en futuras investigaciones la sociedad y los juegos desde la 
Andújar, I., y Brasó, J. (2017). La lógica interna en Los Juegos de niños (1560) de Peter Brueghel. RICYDE. Revista internacional de ciencias del deporte, 50(13), 426-441.

multitud de otros aspectos posibles, con el fin de poder interpretar la imagen de la sociedad de una época.

Asimismo, el análisis de los Juegos de los niños puede ser motivo de recuperación de la memoria histórica. Y quizá, la mejor manera de no olvidar estos juegos consista en enseñarlos a los niños. Por lo tanto, habrá que concretar y desarrollar, en un siguiente paso, posibles intervenciones didácticas, principalmente desde el área de la educación física.

\section{Referencias}

Álvarez-Uría, J., y Varela, J. (1991). Arqueología de la escuela. Madrid: Ed. La Piqueta.

Althusser, L. (1974). Escritos. Barcelona: Laia.

Althusser, L. (1989). La filosofía como arma de la revolución. México: Siglo XXI.

Amades, J. (1947). Auca dels jocs de la mainada. Barcelona: Tallers Hesperia.

Ariès, P. (1960). L'enfant et la vie familiale sous l'Ancien Régime. París: Plon.

Balius, R. (2008). Nuestra portada. Juegos Infantiles. Apunts: Educación Física y Deportes, 3(93), 79-81.

Bennassar, B., Jacquart, J., Lebrun, F., Denís, M., y Blayau, N. (2005). Historia Moderna. Madrid: Akal.

Brasó, J., y Collell, X. (2016). El joc popular de la xarranca. Estudi a través dels llibres i del folklore català. Possibles aplicacions en l'àmbit escolar. REIRE. Revista d'Innovació $i$ Recerca en Educació, 82-105. Disponible en:

http://www.raco.cat/index.php/REIRE/article/view/311704 [Consulta: 3 de febrero de 2017]

Brasó, J., y Torrebadella, X. (2015). Anàlisi i classificació dels jocs de la infància de Joan Amades en funció de la seva lògica interna i del gènere dels practicants (1674-1947). REIRE. Revista d'Innovació i Recerca en Educació, 8(2), 18-42. Disponible en: http://www.raco.cat/index.php/REIRE/article/view/297639 [Consulta: 3 de febrero de 2017]

Breyer, C. (2010). Jeux et jouets à travers les àges. Bruselas: Safran.

Calmels, D. (2007). Juegos de Crianza. Buenos Aires: Biblos.

Cámara, A., y Carrió-Invernizzi, D. (2015). Historia del Arte de los siglos XVII y XVIII. Madrid: UNED.

Carlo, G. (1998). El arte moderno. Madrid: Akal.

Carmona, R. (2009). Juegos tradicionales, patrimonio cultural inmaterial de la humanidad. Una revisión a través de la pintura. EmásF. Revista Digital de Educación Física, 3(15), 7-20. Castelli, E. (2007). Lo demoníaco en el arte: su significado filosófico. Madrid: Siruela.

Charles, V., Manca, J., McShane, M., y Wigal, D. (2006). 1000 Pinturas de los Grandes Maestros. México: Númen.

Córdoba, A., Herrador, J., Martínez, F., y Bueno, J. (2011). VII Congreso Nacional de Ciencias del deporte y educación Física. Alicante: Alto Rendimiento.

Cruzada, D. (1870). Los tapices de Goya. Madrid: Rivandeneyra.

Departamento de Educación. (2005). Las técnicas artísticas. Madrid: Museo ThyssenBornemisza / Akal. 
Andújar, I., y Brasó, J. (2017). La lógica interna en Los Juegos de niños (1560) de Peter Brueghel. RICYDE. Revista internacional de ciencias del deporte, 50(13), 426-441.

Díaz, M., y Mango, E. (2011). Propuestas en torno a la obra de Brueghel: "Juegos infantiles". Disponible en:

http://uruguayeduca.edu.uy/Portal.Base/Web/VerContenido.aspx?ID=210181 [Consulta: 3 de febrero de 2017]

Elias, N. y Dunning, N. (1992) Deporte y ocio en el proceso de la civilización. México: Fondo de Cultura Económica.

Elschenbroich, D. (1979). El juego de los niños. Estudios sobre la génesis de la infancia. Bilbao: Zero.

Frascina, F., Blake, N., Fer, B., Garb, T., y Harrison, C. (1998). La modernidad y lo moderno. Madrid: Akal.

García, A., y Llull, J. (2009). El juego infantil y su metodología. Madrid: Editex.

García, B. (1999). El Ocio en la España del Siglo de Oro. Madrid: Akal.

Gómez, I. (2009). La variedad del mundo. Madrid: Siruela.

González Aja, T. (1990). Actividades lúdicas en Goya. Revista de Investigación y Documentación sobre las Ciencias de Educación Física y del Deporte, 15-16, 93-100.

González Aja, T. (1992). El deporte a través del arte Occidental [tesis doctoral]. Madrid: Universidad Complutense de Madrid.

González Aja, T. (1988). Les Jeux dans la peinture de Goya. Sports, arts et religion. Larcana: C.R. Staps Paris.

González, J. (1993). Tractatus Ludorum: una antropología del juego. Barcelona: Antrophos.

Gracia, L. (1978). Juegos tradicionales aragoneses. Zaragoza: Libreria General.

Grosvenor, I. (2007) From the 'Eye of History' to 'a Second Gaze': The visual archive and the marginalized in the history of eduction. History of Education 36(4), 607-622.

https://doi.org/10.1080/00467600701496948

Grosvenor, I. (2016) From Voices of War to Voices of Peace: exploring the legacy of the First World War through participatory research. Pensament pedagògic $i$ Discursos Educatius en la Construcció Europea cent anys després de la Gran Guerra $1^{a}$ Jornada Internacional Recercaixa. Divendres 25 de novembre de 2016. Barcelona: Departament de Teoria i Història de I'Educació (Universitat de Barcelona).

Herrador, J. (2005). Juegos populares y tradicionales en Pintura Flamenca de P. Brueguel (S.XVI): una propuesta en el área de la Educación Física. Revista de educación física: renovar la teoría y práctica, 3(99), 21-36.

Herrador, J. (2012). Análisis de los juegos populares de P. Brueguel a través de las vitolas. $A D A L, 24,25-29$.

Herrador, J. (2013). Juegos tradicionales en la pintura plasmada en los sellos postales. Movimento humano, 1(5), 11-31.

Herrador, J., Zagalaz, M., Martínez, E., y Rodríguez, I. (2006). Actividades lúdicotradicionales en la pintura flamenca de P. Brueguel (s. XVI). En Alto Rendimiento (Ed.), I Congreso Internacional de las Ciencias Deportivas (p. 1). Alicante: Alto Rendimiento.

Hills, J. (1940). Children games by Pieter Brugel the elder: a folkloristic investigation. Chicago: Universidad de Chicago.

Hills, J. (1956). Das Kinderspielbild Pieter Bruegel d. Aelter [tesis]. Viena: s.e.

Lagardera, F. y Lavega, P. (2003). Introducción a la praxiología motriz. Barcelona: Paidotribo. 
Andújar, I., y Brasó, J. (2017). La lógica interna en Los Juegos de niños (1560) de Peter Brueghel. RICYDE. Revista internacional de ciencias del deporte, 50(13), 426-441.

Lavega, P., y Navarro, V. (2014) La motricidad en los juegos de Rodrigo Caro: Días geniales o lúdricos (1626). Revista Internacional de Medicina y Ciencias de la Actividad Física y el Deporte, 15 (59), 489-505. Disponible en:

http://cdeporte.rediris.es/revista/revista59/artmotricidad616.htm [Consulta: 15 de junio de 2017]

López, F. (2013). Los pájaros y el fantasma: una historia del artista en el paisaje. Salamanca: Ediciones Universidad.

Manzano, P. (2014). Los juegos tradicionales de Pieter Bruegel. Disponible en: http://webquestpieterbruegel.weebly.com/ [Consulta: 10 de diciembre de 2016]

Mateo, I. (1991) El Bosco en España. Madrid: CSIC.

Mateo, I. (1959). El grupo de jugadores en 'El Jardín de las Delicias', del Bosco. Archivo Español de Arte, 32127, 252.

McNeill, W. (2000). La Civilización de Occidente: Manual de Historia. Puerto Rico: Editorial de la Universidad de Puerto Rico.

Mehl, J. (1990). Les jeux au royaume de France: du XIIIe au début du XVIe siècle. París: Fayard.

Mena, M. (2000). Goya: guía de la sala. Madrid: Alianza.

Menzel, G. (1966). Pieter Brueghel der Aelter. Leipzig: s.e.

Mestre, J. (1973). Goya o los Juegos y Recreos de una Sociedad Española. Madrid: Deporte 2000.

Navacerrada, R. (2007). Jeux d'enfants: una visión detallada del juego tradicional en el Renacimiento europeo. Lecturas: Educación Física y Deportes, 12(115), 1. Disponible en: http://www.efdeportes.com/efd115/jeux-d-enfants-juego-tradicional-en-elrenacimiento.htm [Consulta: 10 de marzo de 2017]

Navacerrada, R. (2010). Juegos olvidados, juegos recuperados.... Revista Española de Educación Física y Deportes, 391, 13-28. Disponible en:

http://www.reefd.es/index.php/reefd/article/view/251/242 [Consulta: 20 de mayo de 2017]

Navacerrada, R. (2012). El mundo lúdico en la literatura de Rabelais: La obra Gargantua y el repertorio de juegos del capítulo XXII de la edición 1542 [tesis doctoral no publicada]. Madrid: INEF.

Parlebas, P. (2001). Juegos, deporte y sociedades. Léxico de praxeología motriz. Barcelona: Paidotribo.

Parlebas, P. (2003). Le destin des jeux: Héritage et filiation. Socio-Anthropologie, 1(13), $1-9$.

https://doi.org/10.4000/socio-anthropologie.173

Pavón, C., Herrador, J., Sánchez, A., y Márquez, I. (2011). Juegos de niños en la pintura de P. Brueghel. Análisis de diferentes soportes iconográficos. En VII Congreso Nacional de Ciencias del Deporte y Educación Física. Sevilla: Universidad Pablo de Olavide.

Pelegrín, A. (1992). Juegos y poesía popular en la literatura infantil y juvenil (17501987) [tesis doctoral]. Madrid: Universidad Complutense de Madrid. Facultad de Filología.

Pelegrín, A. (1998). Repertorio de antiguos juegos infantiles: tradición y literatura hispánica. Madrid: CSIC.

Pérez Vejo, T. (2012). ¿Se puede escribir historia a partir de imágenes? El historiador y las fuentes icónicas. Memoria y sociedad, 16(32), 17-30. 
Andújar, I., y Brasó, J. (2017). La lógica interna en Los Juegos de niños (1560) de Peter Brueghel. RICYDE. Revista internacional de ciencias del deporte, 50(13), 426-441.

Pérez, M., y Benet, R. (2014). Jocs d'Infants. Ferran Casablancas. Disponible en: https://youtu.be/pxkMRLOhASk [Consulta: 10 de diciembre de 2016]

Portmann, P. (1961). Die Kinderspiele Pieter Brueghel d. A. Berlín: Hallwag.

Prohaska, W. (2004). El Kunsthistorisches Museum de Viena. La Pinacoteca. Londres: Scala.

Pubill, B. (2009). De Bruegel a la Play. Un viaje de 450 años a través de los juegos. Tándem, 1(29), 1-6.

Redondo, E. y Laspalas, J. (1997). Historia de la Educación. Edad Antigua. Madrid: Dykinson.

Riaño, P. (2013). La otra Gioconda: El reflejo de un mito. Madrid: Debate.

Riordan, J., y Krüger, A. (2003). European Cultures of Sport. Examining the Nations and Regions. Bristol: Intellect.

Saccà, I. [dir]. (2011). Enciclopedia Moderna. Enciclopedia Britannica. Santiago de Chile: Sociedad Comercial.

Soto, J. (2010). Los Hyeroglyphica en "El asno en la escuela" de Brueghel. Revista Sans Soleil - Estudios de la Imagen, 1(2), 55-58.

Stella, J. (1657). Les jeux et plaisiris de l'enfance. Paris: Aux galleries du Louvre chez la ditte Stella.

Stella, J. (1990). Juegos y pasatiempos de la infancia. Palma de Mallorca: José J. de Olañeta.

Strosetzki, C. (1996). Ocio, trabajo y juego. Aspectos de su valoración en algunos tratados del siglo de oro. Siglo de Oro. Actas del IV congreso internacional de la AISO, 2, 1547-1553.

Torrebadella, X., y Brasó, J. (2014) El juego del marro. Análisis de la lógica interna y posibilidades educativas, Tándem. Didáctica de la Educación Física, 45, 75-79.

Torrebadella, X. (2009). Contribución a la historia de la educación física en España. Estudio bio-bibliográfico en torno a la educación física y el deporte (1800-1939) [tesi doctoral no publicada]. Lleida: Universitat de Lleida.

Torrebadella, X. (2011). Repertorio bibliográfico inédito de la educación física y el deporte en España (1800-1939). Madrid: Fundación Universitaria Española.

Torrebadella, X. (2016). Quan l'educació física arriba a l'escola primària. Notes per a la història de l'educació a Lleida (1901-1931). Educació i Història: Revista d'Història de I'Educació, 2(28), 255-287.

Turcot, L. (2016). Sports et Loisirs. Une historie des origines à nos jours. Paris: Gallimard.

Universidad Pablo de Olavide. (4 de junio de 2013). Escenificación de 'Juego de niños' de Pieter Brueghel. Disponible en: https://youtu.be/rCuUOGvAHsM [Consulta: 10 de diciembre de 2016]

Varela, J. (1986). Aproximación genealógica a la moderna percepción social de los niños. Revista de Educación, 281, 155-175.

Vanden, J. (1982). En P. Ariès y J. Margolin, Les jeux à la Reannaissance. Actes du XXIII Colloque International d'études Humanistes (pp. 499-524). Paris: Librairie Philosophique J. Vrin.

Zambrano, J. (2012). Acercamiento interartístico a la pintura de género de Murillo y el pícaro literario del Siglo de Oro. Personajes que reflejan una realidad social. Ontario: Universidad Western. 